\title{
Efficacy of GAD-alum immunotherapy associated with HLA-DR3-DQ2 in recently diagnosed type 1 diabetes
}

\author{
Ulf Hannelius $^{1}$ (D) $\cdot$ Craig A. Beam ${ }^{2}$ (i) $\cdot$ Johnny Ludvigsson ${ }^{3,4}$ (D) \\ Received: 28 April 2020 / Accepted: 11 June 2020 / Published online: 5 August 2020 \\ (C) The Author(s) 2020
}

\begin{abstract}
Aims/hypothesis The aim of this study was to determine if retention of C-peptide following immunotherapy using recombinant GAD65 conjugated to aluminium hydroxide (GAD-alum) is influenced by HLA risk haplotypes $D R 3-D Q 2$ and $D R 4-D Q 8$. Methods HLA-dependent treatment effect of GAD-alum therapy on C-peptide retention in individuals with recent-onset type 1 diabetes was evaluated using individual-level patient data from three placebo-controlled, randomised clinical trials using a mixed repeated measures model.

Results A significant and dose-dependent effect was observed in individuals positive for the genotypes that include HLA-DR3$D Q 2$ but not $H L A-D R 4-D Q 8$ and in the broader subgroup of individuals positive for all genotypes that include $H L A-D R 3-D Q 2$ (i.e. including those also positive for $H L A-D R 4-D Q 8)$ ). Higher doses (three or four injections) showed a treatment effect ratio of 1.596 (95\% CI 1.132, 2.249; adjusted $p=0.0035)$ and 1.441 (95\% CI 1.188, 1.749; adjusted $p=0.0007)$ vs placebo for the two respective HLA subgroups.

Conclusions/interpretation GAD65-specific immunotherapy has a significant effect on C-peptide retention in individuals with recent-onset type 1 diabetes who have the $D R 3-D Q 2$ haplotype.
\end{abstract}

Keywords Antigen-specific · Autoimmune diabetes · C-peptide · GAD · Glutamic acid decarboxylase · HLA · Immunotherapy · Type 1 diabetes $\cdot$ Vaccine

\section{Abbreviation \\ GAD-alum Recombinant human GAD65 conjugated to aluminium hydroxide \\ rhGAD65 Recombinant human GAD65}

Ulf Hannelius

ulf.hannelius@diamyd.com

$\triangle$ Johnny Ludvigsson

Johnny.Ludvigsson@liu.se;

Johnny.Ludvigsson@ @egionostergotland.se

1 Diamyd Medical AB, Kungsgatan 29, 11156 Stockholm, Sweden

2 Department of Biomedical Sciences, Western Michigan University Homer Stryker M.D. School of Medicine, Kalamazoo, MI, USA

3 Crown Princess Victoria Children's Hospital, Linköping, Sweden

4 Division of Pediatrics, Department of Biomedical and Clinical Sciences (BKV), Medical Faculty, Linköping University, SE 58185 Linköping, Sweden

\section{Introduction}

Recombinant human GAD65 conjugated to aluminium hydroxide (GAD-alum) is an antigen-specific immunotherapy intended to induce specific immunological tolerance to preserve the pancreatic beta cells that are targeted in type 1 diabetes by autoreactive cytotoxic $\mathrm{T}$ cells. The active ingredient, recombinant human GAD65, is a pancreatic beta cell protein, GAD65 being one of the most frequent autoantigens associated with type 1 diabetes. The effect of GAD-alum on preserving endogenous insulin production has been evaluated in several placebo-controlled, randomised trials in individuals recently diagnosed with type 1 diabetes, albeit with inconclusive results [1-4]. It is becoming increasingly clear that factors such as genetic background in the form of HLA genotype affect both the risk of diabetes affliction and the pathogenesis of the disease [5-13]. It is conceivable that HLA may also influence the effect of antigen-specific immunotherapies like GAD-alum and while this possibility has been considered incidentally in prior clinical trials $[2,3]$, this hypothesis has not been extensively evaluated. 


\section{Research in context}

\section{What is already known about this subject?}

- HLA affects the risk of type 1 diabetes and autoantibody seroconversion

- $\quad H L A-D R 3-D Q 2$ associates with GAD65 autoimmunity

- GAD65-specific immunotherapy has a positive effect on beta cell preservation in type 1 diabetes

\section{What is the key question?}

- Does HLA genotype influence the effect of therapy with recombinant human GAD65 conjugated to aluminium hydroxide (GAD-alum)?

What are the new findings?

- HLA genotype significantly influences the effect of GAD-alum therapy

- The best effect of GAD-alum is seen in individuals positive for HLA DR3-DQ2 but negative for HLA-DR4-DQ8

How might this impact on clinical practice in the foreseeable future?

- Stratifying individuals based on HLA risk haplotypes may considerably speed up the development and introduction of safe and effective antigen-specific and other immunomodulatory therapies for type 1 diabetes

The aim of this study was to estimate, using individuallevel patient data from previous randomised, placebocontrolled trials, whether the efficacy of GAD-specific immunotherapy depends on the presence of the GAD and insulin antibody-associated HLA haplotypes $D R 3-D Q 2$ and $D R 4$ $D Q 8$.

\section{Methods}

We combined individual-level data from three published randomised, controlled clinical trials [2-4], clinical trial identifiers NCT00435981, NCT00529399 and NCT00723411, that evaluated subcutaneous GAD-alum therapy (compared with alum) in GAD autoantibody-positive individuals with recent-onset type 1 diabetes.

The similarities and differences between the clinical studies have been described in detail elsewhere [1]. Table 1 summarises the number of participants eligible for the analysis, treatment schedules and HLA genotype distribution. Briefly, all trials evaluated treatment with two injections of $20 \mu \mathrm{g}$ GADalum or alum only (placebo). Two trials [2, 3] also evaluated three or four injections of $20 \mu \mathrm{g}$ GAD-alum. GAD-alum or placebo was administered at days 1, 30, 90 and 270, respectively. For the analysis, the treatment was coded as placebo, low dose (two injections) and high dose (three or four injections).

A total of 521 participants (of 549) were included in the final analysis. Patients that did not have a baseline and at least one post-baseline C-peptide value ( $n=12$ out of 549) were excluded as well as individuals with missing HLA information ( $n=16$ out of 537 remaining). The HLA subgroup term was coded as either presence or absence of genotypes that include $H L A-D R 3-D Q 2$ and, in a second model, as either presence or absence of genotypes that include $H L A-D R 3-$ $D Q 2$ but not HLA-DR4-DQ8.

The treatment effect on C-peptide retention $\left(\log _{e}\right.$ of the ratio of C-peptide AUC at 15 months/C-peptide AUC at baseline) was estimated using the restricted maximum likelihood approach in a mixed repeated measures model. All measurements from baseline to the primary endpoint readout (12 months for NCT00529399, 15 months for NCT00435981 and NCT00723411) were used. The model was adjusted for the fixed effects of baseline C-peptide, study, treatment, HLA subgroup, visit, country, sex and age, as well as the interaction of baseline $\mathrm{C}$-peptide by visit and treatment by HLA subgroup by visit. Baseline value, age and visit were treated as continuous variables. Study, treatment, HLA subgroup, country and sex were treated as categorical variables. Patient identification number and country were included as categorical random effects to yield a variance components structure. The treatment effect ratios at 15 months were based on least square means with adjusted $p$ values using the Bonferroni-ŠSidák correction reported along with two-sided $95 \%$ confidence intervals. A $p$ value of $<0.05$ was considered significant. Statistical analysis was performed using SAS version 9.4 (SAS Institute, Cary, NC, USA).

\section{Results}

A significant interaction was observed in the main statistical model between treatment, $\mathrm{C}$-peptide retention $\left(\log _{e}\right.$ of the $\mathrm{C}$ peptide AUC ratio 15 months/baseline), and the HLA-DR3$D Q 2(p=0.02)$ or $D R 3-D Q 2 /$ not $D R 4-D Q 8$ genotype groups 
Table 1 Summary of data, HLA genotype distribution and treatment regimens as evaluated in the analysis

\begin{tabular}{|c|c|c|c|}
\hline & $\begin{array}{l}\text { Ludvigsson et al, } 2008 \\
\text { (NCT00435981) [4] }\end{array}$ & $\begin{array}{l}\text { Wherrett et al, 2011 } \\
\text { (NCT00529399) [3] }\end{array}$ & $\begin{array}{l}\text { Ludvigsson et al, } 2012 \\
\text { (NCT00723411) [2] }\end{array}$ \\
\hline Participants eligible for analysis, $n$ & 69 & 139 & 313 \\
\hline All participants & 70 & 145 & 334 \\
\hline $\begin{array}{l}\text { With baseline and at least one } \\
\text { post-baseline value missing }\end{array}$ & 1 & 4 & 7 \\
\hline With missing HLA information & 0 & 2 & 14 \\
\hline \multicolumn{4}{|l|}{ HLA distribution, $n(\%)$} \\
\hline$H L A-D R 3-D Q 2$ & $34(49 \%)$ & $71(50 \%)$ & $161(50 \%)$ \\
\hline$H L A-D R 3-D Q 2 / \operatorname{not} H L A-D R 4-D Q 8$ & $17(24 \%)$ & $36(25 \%)$ & $74(23 \%)$ \\
\hline \multicolumn{4}{|l|}{ Treatment schedule $^{\mathrm{a}}$} \\
\hline Low dose & $\begin{array}{l}\text { Two doses of } 20 \mu \mathrm{g} \\
\text { GAD-alum (days } 1 \text { and } 30)\end{array}$ & $\begin{array}{l}\text { Two doses of } 20 \mu \mathrm{g} \text { GAD-alum } \\
\text { (days } 1 \text { and } 30 \text { ), one dose of } \\
\text { alum (day 90) }\end{array}$ & $\begin{array}{l}\text { Two doses of } 20 \mu \mathrm{g} \text { GAD-alum } \\
\text { (days } 1 \text { and } 30 \text { ), two doses of } \\
\text { alum (days } 90 \text { and 180) }\end{array}$ \\
\hline High dose & - & $\begin{array}{l}\text { Three doses of } 20 \mu \mathrm{g} \text { GAD-alum } \\
\quad \text { (days } 1,30 \text { and } 90 \text { ) }\end{array}$ & $\begin{array}{l}\text { Four doses of } 20 \mu \mathrm{g} \text { GAD-alum } \\
\quad(\text { days } 1,30,90 \text { and } 180)\end{array}$ \\
\hline Placebo & $\begin{array}{l}\text { Two doses of alum } \\
\text { (days } 1 \text { and } 30 \text { ) }\end{array}$ & $\begin{array}{l}\text { Three doses of alum (days 1, } 30 \\
\text { and 90) }\end{array}$ & $\begin{array}{l}\text { Four doses of alum (days } 1,30, \\
90 \text { and } 180 \text { ) }\end{array}$ \\
\hline
\end{tabular}

${ }^{\text {a }}$ For Wherrett et al 2011 the treatment schedule was defined as baseline, week 4 and week 12, while Ludvigsson 2008 and 2012 defined the schedule based on days from baseline $(1,30,90$ and 180). For consistency, days from baseline are used in the table. For a detailed description on the similarities and differences between the clinical trials, please see [1]

$(p=0.03)$, indicating that the treatment effect of GAD-alum differs depending on the patient's HLA haplotype. Post hoc tests showed that the estimated treatment effect ratio (Fig. 1) of GAD-alum compared with placebo at 15 months from baseline was 1.318 (95\% CI 1.124, 1.545; adjusted $p=$ $0.0007)$ in individuals positive for $D R 3-D Q 2(n=266)$ genotype and 1.401 (95\% CI 1.109, 1.769; adjusted $p=0.0047)$ in individuals positive for the $D R 3-D Q 2 /$ not $D R 4-D Q 8$ ( $n=$ 127) genotypes. A higher dose (three or four injections of GAD-alum) showed a treatment effect ratio of 1.441 (95\% CI $1.188,1.749$; adjusted $p=0.0007)$ and $1.596(95 \% \mathrm{CI}$ $1.132,2.249$; adjusted $p=0.0035)$ vs placebo for the two HLA subgroups. No significant effect after adjusting for multiple testing was seen in these subgroups following a lower dose (two injections).

Fig. 1 Estimated treatment effect ratio (C-peptide retention; active vs placebo) at 15 months post baseline in patients with or without genotypes that include $H L A-D R 3-D Q 2(n=266$ and 255), or HLA-DR3-DQ2 but not HLA-DR4-DQ8 ( $n=127$ and $n=$ 394). Adjusted $p$ values using the Bonferroni- Šidák correction are reported along with two-sided $95 \%$ CIs

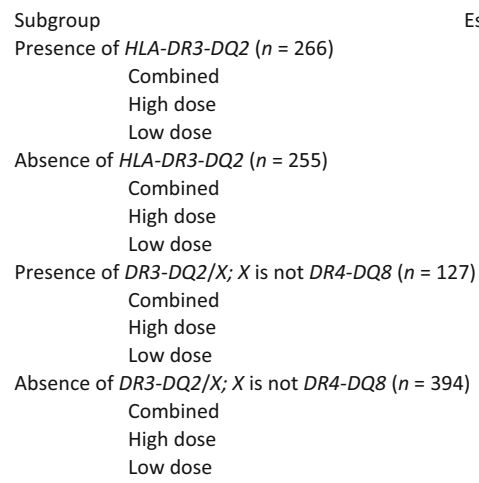

In individuals negative for the $D R 3-D Q 2 /$ not $D R 4-D Q 8$ genotypes no significant effect was seen for any dose or for the combination of doses. For individuals negative for $D R 3$ $D Q 2$ genotypes, a treatment effect ratio of 1.203 (95\% CI 1.004, 1.440; adjusted $p=0.043$ ) for lower dose was seen. No significance was seen for higher dose or for the combined dose regimen.

\section{Discussion}

This study demonstrates that the efficacy of GAD-alum immunotherapy may depend on specific HLA risk haplotypes. The concept that HLA molecules play an important role in guiding antigen-specific autoimmunity in type 1 diabetes is 
not new as previous studies demonstrate that the first appearance of either GAD or insulin autoantibodies was associated with $H L A-D R 3-D Q 2$ and $D R 4-D Q 8$, respectively [7]. However, this is the first report that we are aware of identifying the ability of specific HLA types to determine the efficacy of GAD-based therapeutics.

Specifically, based on this analysis where we combine individual patient-level data from three previous placebocontrolled clinical trials, we show that GAD-alum immunotherapy has a significant and dose-dependent effect on Cpeptide retention in individuals positive for genotypes that include the HLA-DR3-DQ2 haplotype. We further show that the effect is most pronounced in individuals who are simultaneously negative for genotypes that include the HLA-DR4$D Q 8$ haplotype. Interestingly, recent research highlights the existence of different phenotypes of type 1 diabetes based on autoantibody seroconversion that, in turn, are linked to HLA risk haplotypes [7, 9, 10]. More specifically and as noted above, as HLA-DR3-DQ2 has been associated with seroconversion to GAD65 autoantibodies and $D R 4-D Q 8$ with seroconversion to insulin autoantibodies $[7,10]$, our results suggest that the best efficacy of antigen-specific immunotherapy may be achieved when targeting individuals that show a specific HLA type that is linked to the tolerising antigen.

These findings are in alignment with recent discussions highlighting the importance of considering disease heterogeneity in type 1 diabetes when evaluating therapeutic strategies [10-13]. Importantly, HLA genotyping is a straightforward and clinically feasible strategy to prospectively identify individuals with type 1 diabetes that have a higher likelihood of responding to the GAD-alum treatment.

Going forward, HLA information will be an integral part of any type 1 diabetes trial with GAD-alum. The current retrospective analyses involve comprehensive data from three randomised controlled trials conducted both in Europe and in the USA, with slightly different inclusion criteria. As these analyses were performed post hoc, it will be important to prospectively verify the findings presented here with regards to the interaction of HLA and GAD-alum therapy in ongoing trials. It will also be important to understand how HLA affects the immunological response to the therapy. Interestingly, preliminary findings (U. Hannelius, M. Danelljan, unpublished results) indicate that HLA does affect the GAD antibody response. There is also a clear rationale to retrospectively analyse the interaction between HLA and treatment in other antigen-specific trials, especially where insulin has been used as the tolerising antigen given the association between insulin autoimmunity and HLA-DR4-DQ8.

Acknowledgements We thank M. Atkinson, University of Florida, and A. Lernmark, Lund University, for valuable comments on the manuscript.
Funding Open access funding provided by Linköping University. This research received no specific grant from any funding agency in the public, commercial or not-for-profit sectors. Diamyd Medical AB was involved in study design and data collection only and did not impose any restrictions regarding the publication of the report.

Data availability The data that support the findings of this study are available on reasonable request from the corresponding author (UH).

Duality of interest $\mathrm{UH}$ is an employee of and owns stock in Diamyd Medical. CAB has served in a consulting role for Diamyd Medical AB. $\mathrm{JL}$ has received an unrestricted research grant from Diamyd Medical AB.

Contribution statement UH designed the study, analysed the data and wrote the manuscript. $\mathrm{CAB}$ contributed to design of the study, analysed the data and reviewed/edited the manuscript. JL contributed to the design of the study and reviewed/edited the manuscript. All authors approved the final version of the manuscript. UH is the guarantor of this work and, as such, had full access to all the data in the study and takes responsibility for the integrity of the data and the accuracy of the data analysis. UH attests that all listed authors meet authorship criteria and that no others meeting the criteria have been omitted. Diamyd Medical AB was not involved in the analysis and interpretation of data or in writing the report.

Open Access This article is licensed under a Creative Commons Attribution 4.0 International License, which permits use, sharing, adaptation, distribution and reproduction in any medium or format, as long as you give appropriate credit to the original author(s) and the source, provide a link to the Creative Commons licence, and indicate if changes were made. The images or other third party material in this article are included in the article's Creative Commons licence, unless indicated otherwise in a credit line to the material. If material is not included in the article's Creative Commons licence and your intended use is not permitted by statutory regulation or exceeds the permitted use, you will need to obtain permission directly from the copyright holder. To view a copy of this licence, visit http://creativecommons.org/licenses/by/4.0/.

\section{References}

1. Beam CA, MacCallum C, Herold KC, Wherrett DK, Palmer J, Ludvigsson J (2017) GAD vaccine reduces insulin loss in recently diagnosed type 1 diabetes: findings from a Bayesian meta-analysis. Diabetologia 60(1):43-49. https://doi.org/10.1007/s00125-0164122-1

2. Ludvigsson J, Krisky D, Casas R et al (2012) GAD65 antigen therapy in recently diagnosed type 1 diabetes mellitus. $\mathrm{N}$ Engl $\mathrm{J}$ Med 366(5):433-442. https://doi.org/10.1056/NEJMoa1 107096

3. Wherrett DK, Bundy B, Becker DJ et al (2011) Antigen-based therapy with glutamic acid decarboxylase (GAD) vaccine in patients with recent-onset type 1 diabetes: a randomised doubleblind trial. Lancet 378(9788):319-327. https://doi.org/10.1016/ S0140-6736(11)60895-7

4. Ludvigsson J, Faresjo M, Hjorth M et al (2008) GAD treatment and insulin secretion in recent-onset type 1 diabetes. N Engl J Med 359(18):1909-1920. https://doi.org/10.1056/NEJMoa0804328

5. Ziegler AG, Nepom GT (2010) Prediction and pathogenesis in type 1 diabetes. Immunity 32(4):468-478. https://doi.org/10.1016/j. immuni.2010.03.018

6. Krischer JP, Liu X, Lernmark A et al (2017) The influence of type 1 diabetes genetic susceptibility regions, age, sex, and family history on the progression from multiple autoantibodies to type 1 diabetes: A TEDDY study report. Diabetes 66(12):3122-3129. https://doi. org/10.2337/db17-0261 
7. Krischer JP, Lynch KF, Lernmark A et al (2017) Genetic and environmental interactions modify the risk of diabetes-related autoimmunity by 6 years of age: The TEDDY study. Diabetes Care 40(9): 1194-1202. https://doi.org/10.2337/dc17-0238

8. Schenker M, Hummel M, Ferber K et al (1999) Early expression and high prevalence of islet autoantibodies for DR3/4 heterozygous and DR4/4 homozygous offspring of parents with type I diabetes: the German BABYDIAB study. Diabetologia 42(6):671-677. https://doi.org/10.1007/s001250051214

9. Krischer JP, Lynch KF, Schatz DA et al (2015) The 6 year incidence of diabetes-associated autoantibodies in genetically at-risk children: the TEDDY study. Diabetologia 58(5):980-987. https:// doi.org/10.1007/s00125-015-3514-y

10. Battaglia M, Ahmed S, Anderson MS et al (2020) Introducing the endotype concept to address the challenge of disease heterogeneity in type 1 diabetes. Diabetes Care 43(1):5-12. https://doi.org/10. 2337/dc19-0880
11. Arif S, Leete P, Nguyen V et al (2014) Blood and islet phenotypes indicate immunological heterogeneity in type 1 diabetes. Diabetes 63(11):3835-3845. https://doi.org/10.2337/db14-0365

12. Claessens LA, Wesselius J, van Lummel M et al (2020) Clinical and genetic correlates of islet-autoimmune signatures in juvenileonset type 1 diabetes. Diabetologia 63(2):351-361. https://doi.org/ 10.1007/s00125-019-05032-3

13. Roep BO, Wheeler DCS, Peakman M (2019) Antigen-based immune modulation therapy for type 1 diabetes: the era of precision medicine. Lancet Diabetes Endocrinol 7(1):65-74. https://doi.org/ 10.1016/S2213-8587(18)30109-8

Publisher's note Springer Nature remains neutral with regard to jurisdictional claims in published maps and institutional affiliations. 